Bull. Austral. Math. Soc.

20D10, 20F 17

VOL. 68 (2003) [461-470]

\title{
A QUESTION FROM THE KOUROVKA NOTEBOOK ON FORMATION PRODUCTS
}

\section{A. Ballester-Bolinches, Clara Calvo and R. Esteban-Romero}

It is shown in this paper that if $\mathfrak{X}$ is a class of simple groups such that $\pi(\mathfrak{X})=\operatorname{char} \mathfrak{X}$, the $\mathfrak{X}$-saturated formation $\mathfrak{H}$ generated by a finite group cannot be expressed as the Gaschütz product $\mathfrak{F} \circ \mathfrak{G}$ of two non- $\mathfrak{X}$-saturated formations if $\mathfrak{H} \neq \mathfrak{G}$. It answers some open questions on products of formations. The relation between $\omega$-saturated and $\mathfrak{X}$-saturated formations is also discussed.

\section{INTRODUCTION}

All groups considered in this paper are tacitly assumed to be finite.

Recall that a formation $\mathfrak{F}$ is a class of groups which is closed under taking homomorphic images and subdirect products. It is known that, if $\mathfrak{F}$ is a formation, each group $G$ has a smallest normal subgroup whose factor group is in $\mathfrak{F}$. Such subgroup is called the $\mathfrak{F}$-residual of $G$ and it is denoted by $G^{\mathfrak{F}}$. A formation $\mathfrak{F}$ is said to be saturated if $G \in \mathfrak{F}$ whenever the Frattini factor group $G / \Phi(G)$ is in $\mathfrak{F}$. A local approach to saturation is $p$-saturation, where $p$ is a prime number. We say that a formation $\mathfrak{F}$ is $p$-saturated if $G \in \mathfrak{F}$ provided the factor group $G /\left(\mathrm{O}_{p}(G) \cap \Phi(G)\right)$ belongs to $\mathfrak{F}$. It is clear that a formation $\mathfrak{F}$ is saturated if and only if $\mathfrak{F}$ is $p$-saturated for all primes $p$. This concept arises spontaneously when the saturation of formation products is considered.

Given two classes $\mathfrak{Y}$ and $\mathfrak{Z}$ of groups, a class product can be defined by setting

$$
\mathfrak{Y} \mathbf{3}:=(G \in \mathfrak{E} \mid \text { there is a normal subgroup } N \text { of } G
$$

$$
\text { such that } N \in \mathfrak{Y} \text { and } G / N \in \mathfrak{Z} \text { ), }
$$

where $\mathfrak{E}$ is the class of all finite groups. This class turns out to be useful in the theory of classes of groups, especially when certain formations are considered. However this class product is not in general a formation when $\mathfrak{Y}$ and $\mathfrak{Z}$ are formations. Fortunately, there is a way of modifying the above definition to ensure that the class product of two formations is again a formation. If $\mathfrak{F}$ and $\mathfrak{G}$ are formations, the formation product or Gaschütz product of $\mathfrak{F}$ and $\mathfrak{G}$ is the class $\mathfrak{F} \circ \mathfrak{G}$ defined by

$$
\mathfrak{F} \circ \mathfrak{G}:=\left(X \in \mathfrak{E} \mid X^{\mathfrak{B}} \in \mathfrak{F}\right) \text {. }
$$

Received 21st May, 2003

Supported by Proyecto BFM2001-1667-C03-03, MCyT (Spain) and FEDER (European Union).

Copyright Clearance Centre, Inc. Serial-fee code: 0004-9727/03 \$A2.00+0.00. 
It is known that $\mathfrak{F} \circ \mathfrak{G}$ is again a formation and if $\mathfrak{F}$ is closed under taking subnormal subgroups, then $\mathfrak{F} \mathfrak{G}=\mathfrak{F} \circ \mathfrak{G}$ (see $[4, I V, 1.7$ and 1.8$]$ ).

The formation product of two formations does not normally yield a saturated formation (see [2]). In fact, if $\mathfrak{F}$ and $\mathfrak{G}$ are formations such that $\mathfrak{F} \circ \mathfrak{G}$ is saturated, then $\mathfrak{G}$ is $p$-saturated for all primes $p$ not dividing the order of any group in $\mathfrak{F}$ (see [9]).

A celebrated theorem, proved by Gaschütz and Lubeseder in the soluble universe and extended by Schmid to the general one, asserts that a formation is saturated if and only if it is local (see $[4$, IV , 4.6]). Partially saturated formations can be characterised by means of a local version of the above result: if $\mathfrak{F}$ is a formation and $p$ is a prime, then $\mathfrak{F}$ is $p$-saturated if and only if $\mathfrak{F}$ is $p$-local, that is, the smallest local formation $\overline{\mathfrak{F}}$ containing $\mathfrak{F}$ is contained in $\mathfrak{N}_{p^{\prime}} \mathfrak{F}$, where $\mathfrak{N}_{p^{\prime}}$ is the class of all nilpotent $p^{\prime}$-groups (see [11]). Moreover, there is a way of constructing the $p$-saturated formations by means of $p$-local definitions (see [3]).

In an unpublished manuscript, Baer gives another extension of the GaschützLubeseder theorem to the general universe. He uses a different concept of local formation and obtains that the so-called Baer formations are exactly the solubly saturated ones (see [4, IV,4.17]). Baer formations were also studied by Shemetkov, who calls them composition formations.

In [7], Skiba asked the following question:

If $\mathfrak{H}=\mathfrak{F} \circ \mathfrak{G}$ is a one-generated Baer formation, where $\mathfrak{F}$ and $\mathfrak{G}$ are non-trivial formations, is $\mathfrak{F}$ a Baer formation?

In the 1999 edition of the same book [8], it is announced that Skiba has answered the question negatively. The reader is referred to [6, p. 224] for a detailed example.

We note that in the known examples of that situation, the equalities $\mathfrak{H}=\mathfrak{B}$ and $\mathfrak{H}=\mathfrak{S}_{p} \mathfrak{H}$ for a prime $p$ hold, where $\mathfrak{S}_{q}$ denotes the class of all $p$-groups. Consequently the following question still remains open:

Assume that $\mathfrak{H}=\mathfrak{F} \circ \mathfrak{G}$ is a Baer formation generated by a group $G$, where $\mathfrak{F}$ and $\mathfrak{G}$ are non-trivial formations. Is $\mathfrak{F}$ a Baer formation provided that $\mathfrak{H} \neq \mathfrak{G}$ or $\mathfrak{H} \neq \mathfrak{S}_{p} \mathfrak{H}$ for every prime $p$ ?

The main result of this paper gives an affirmative answer to that question.

Note that an analogous result was proved by Vishnevskaya in [13] for $p$-saturated formations. She shows that the $p$-saturated formation $\mathfrak{H}$ generated by a finite group cannot be the Gaschütz product $\mathfrak{F} \circ \mathfrak{B}$ of two non- $p$-saturated formations provided $\mathfrak{H} \neq \mathfrak{G}$. This motivates us to present the most general version of our result by using $\mathfrak{X}$-saturated formations, which have been introduced and studied by Förster in [5]; here $\mathfrak{X}$ is a class of simple groups with a completeness property. Although in general there does not exist a class of simple groups $\mathfrak{X}$ such that the $\mathfrak{X}$-saturated formations are exactly the $\omega$-saturated formations (see Example 2 in Section 3), the arguments used in the proof of our result 
still hold for $\omega$-saturated formations. It leads to an alternative proof of Vishnevskaya's result.

THEOREM 1. Let $\mathfrak{X}$ be a class of simple groups such that $\pi(\mathfrak{X})=$ char $\mathfrak{X}$. Let $\mathfrak{H}=\mathfrak{F} \circ \mathfrak{G}$ be an $\mathfrak{X}$-saturated formation generated by a group $G$. If $\mathfrak{F}$ and $\mathfrak{B}$ are non-trivial and either $\mathfrak{H} \neq \mathfrak{G}$ or $\mathfrak{S}_{p} \mathfrak{H} \neq \mathfrak{H}$ for all primes $p \in \operatorname{char} \mathfrak{X}$, then $\mathfrak{F}$ is $\mathfrak{X}$-saturated.

When $\mathfrak{X}=\mathbb{P}$, the class of all Abelian simple groups, the $\mathfrak{X}$-saturated formations are exactly the Baer formations. Therefore we have:

COROLlaRY 1. Let $\mathfrak{H}$ be the Baer formation generated by a group $G$. If $\mathfrak{H}$ $=\mathfrak{F} \circ \mathfrak{G}$ for non-trivial formations $\mathfrak{F}$ and $\mathfrak{G}$, and either $\mathfrak{H} \neq \mathfrak{G}$ or $\mathfrak{S}_{p} \mathfrak{H} \neq \mathfrak{H}$ for all primes $p$, then $\mathfrak{F}$ is a Baer formation.

The above result also seems to be the answer of the Problem 18 of Guo's book [6]. Note that there is an obvious misprint in the statement of that problem, because the example in p. 224 answers that question negatively.

The paper is organised as follows: after collecting some definitions and preliminary results in Section 2, in Section 3 we analyse the relation between $\omega$-saturated formations and $\mathfrak{X}$-saturated formations. The proof of Theorem 1 is presented in Section 4.

For the fundamental concepts of the theory of formations, as well as for the standard notations, the reader is referred to the book of Doerk and Hawkes [4].

\section{PRELIMINARIES}

We begin with the concepts of $\mathfrak{X}$-saturated formation and $\mathfrak{X}$-local formation due to Förster [5]. These concepts arose in order to give a common extension of the theorems of Gaschütz-Lubeseder and Baer.

Denote by $\mathfrak{J}$ the class of all simple groups. For any subclass $\mathfrak{Y}$ of $\mathfrak{J}$, we put $\mathfrak{Y}^{\prime}=\mathfrak{J} \backslash \mathfrak{Y}$. Denote by $\mathrm{E} \mathfrak{Y}$ the class of groups whose composition factors belong to $\mathfrak{Y}$; it is clear that $\mathrm{E} \mathfrak{Y}$ is a Fitting class, and so each group $G$ has a largest normal $E \mathfrak{Y}$-subgroup, the $\mathrm{E} \mathfrak{Y}$-radical $\mathrm{O}_{\mathfrak{Y}}(G)$. A chief factor which belongs to $\mathrm{E} \mathfrak{Y}$ is called a $\mathfrak{Y}$-chief factor, and if, moreover, $p$ divides the order of a $\mathfrak{Y}$-chief factor $H / K$, we shall say that $H / K$ is a $\mathfrak{Y}_{p}$-chief factor.

In the sequel it will be convenient to identify the prime $p$ with the cyclic group $C_{p}$ of order $p$.

Förster's starting point is a class $\mathfrak{X}$ of finite simple groups satisfying

$$
\begin{aligned}
\pi(\mathfrak{X}): & =\{p \in \mathbb{P} \mid \text { there exists } G \in \mathfrak{X} \text { such that } p \text { divides }|G|\} \\
& =\left\{p \in \mathbb{P} \mid C_{p} \in \mathfrak{X}\right\}=: \text { char } \mathfrak{X} .
\end{aligned}
$$

An $\mathfrak{X}$-formation function $f$ associates to each $X \in($ char $\mathfrak{X}) \cup \mathfrak{X}^{\prime}$ a formation $f(X)$ (possibly empty). If $f$ is an $\mathfrak{X}$-formation function, then the $\mathfrak{X}$-local formation $\operatorname{LF}_{\mathfrak{X}}(f)$ defined by $f$ is the class of all groups $G$ satisfying the following two conditions: 
1. if $H / K$ is an $\mathfrak{X}_{p}$-chief factor of $G$, then $G / \mathrm{C}_{G}(H / K) \in f(p)$, and

2. $G / K \in f(E)$ whenever $G / K$ is a monolithic quotient of $G$ such that the composition factor of its $\operatorname{socle} \operatorname{Soc}(G / K)$ is isomorphic to $E$, if $E \in \mathfrak{X}^{\prime}$.

A formation $\mathfrak{F}$ is said to be $\mathfrak{X}$-local if there exists an $\mathfrak{X}$-formation function $f$ such that $\mathfrak{F}=\operatorname{LF}_{\mathfrak{X}}(f)$.

If $\mathfrak{X}=\mathfrak{J}$, the class of all simple groups, an $\mathfrak{X}$-formation function is simply a formation function and the $\mathfrak{X}$-local formations are exactly the local formations. If $\mathfrak{X}=\mathbb{P}$, the class of all Abelian simple groups, an $\mathfrak{X}$-formation function is a Baer function and the $\mathfrak{X}$-local formations are exactly the Baer-local ones (see $[4, \mathrm{IV}, 4.9])$.

Förster also introduced in [5] an $\mathfrak{X}$-Frattini subgroup $\Phi_{\mathfrak{X}}^{*}(G)$ for every group $G$. He defined $\mathfrak{X}$-saturation in the obvious way and he proved that the $\mathfrak{X}$-saturated formations are exactly the $\mathfrak{X}$-local ones. In [1] we introduce another $\mathfrak{X}$-Frattini subgroup in every group $G$, which is smaller than Förster's one.

DEFINITION 1: i. Let $p$ be a prime number. We say that a group $G$ belongs to the class $\mathrm{A}_{\mathfrak{x}_{p}}\left(\mathfrak{P}_{2}\right)$ provided there exists an elementary Abelian normal $p$-subgroup $N$ of $G$ such that

(a) $N \leqslant \Phi(G)$ and $G / N$ is a primitive group with a unique non-abelian minimal normal subgroup, i. e., $G / N$ is a primitive group of type 2 ,

(b) $\operatorname{Soc}(G / N) \in \mathrm{E} \mathfrak{X} \backslash \mathfrak{E}_{p^{\prime}}$, and

(c) $\mathrm{C}_{G}^{h}(N) \leqslant N$, where

$$
\mathrm{C}_{G}^{h}(N):=\cap\left\{\mathrm{C}_{G}(H / K) \mid H / K \text { is a chief factor of } G \text { below } N\right\} .
$$

2. The $\mathfrak{X}$-Frattini subgroup of a group $G$ is the subgroup $\Phi_{\mathfrak{X}}(G)$ defined as

$$
\Phi_{\mathfrak{X}}(G):= \begin{cases}\Phi\left(\mathrm{O}_{\mathfrak{X}}(G)\right) & \text { if } G \notin \mathrm{A}_{\mathfrak{X}_{p}}\left(\mathfrak{P}_{2}\right) \text { for all } p \in \operatorname{char} \mathfrak{X} \\ \Phi(G) & \text { otherwise. }\end{cases}
$$

Note that in the case $\mathfrak{X}=\mathfrak{J}$, the class of all simple groups, $\Phi_{\mathfrak{X}}(G)=\Phi(G)$ for every group $G$, and in the case $\mathfrak{X}=\mathbb{P}$, the class of all Abelian simple groups, $\Phi_{\mathfrak{X}}(G)=\Phi\left(G_{\mathfrak{G}}\right)$, where $G_{\mathfrak{S}}$ is the soluble radical of $G$.

We say that a formation $\mathfrak{F}$ is $\mathfrak{X}$-saturated if $G \in \mathfrak{F}$ provided $G / \Phi_{\mathfrak{X}}(G) \in \mathfrak{F}$.

It is clear then that if $\mathfrak{X}=\mathfrak{J}$, the $\mathfrak{X}$-saturated formations are exactly the saturated ones, and if $\mathfrak{X}=\mathbb{P}$, the $\mathfrak{X}$-saturated formations are just the solubly saturated ones.

We have that $\Phi_{\mathfrak{x}}(G) \leqslant \Phi_{\mathfrak{X}}^{*}(G)$ for every group $G$, but the equality is not true in general (see [1]). However, we have:

THEOREM 2. ([1]) For a formation $\mathfrak{F}$ and a class $\mathfrak{X}$ of simple groups with $\pi(\mathfrak{X})$ $=$ char $\mathfrak{X}$, the following statements are pairwise equivalent:

1. $\mathfrak{F}$ is $\mathfrak{X}$-local, 
2. if $G / \Phi_{\mathfrak{X}}^{*}(G) \in \mathfrak{F}$, then $G \in \mathfrak{F}$, and

3. $\mathfrak{F}$ is $\mathfrak{X}$-saturated.

This result turns out to be crucial in the proof of Theorem 1.

As usual, the $\mathfrak{X}$-saturated formation generated by a group $G$ is the smallest $\mathfrak{X}$ saturated formation containing $G$, that is, the intersection of all $\mathfrak{X}$-saturated formations containing $G$.

We finish the section noting the following fact.

Let $\mathfrak{C}$ be a class of groups. Then there exists a unique smallest $\mathfrak{X}$-saturated formation $\overline{\mathfrak{C}}_{x}$ containing $\mathfrak{C}$, namely the one defined by the $\mathfrak{X}$-formation function $f$ given by

$$
f(X)= \begin{cases}\mathrm{QR}_{0}\left(G / \mathrm{C}_{G}(H / K) \mid G \in \mathfrak{C}, H / K \mathfrak{X}_{p} \text {-chief factor of } G\right) \\ & \text { if } X=p \in \pi(\mathfrak{X}), \\ \mathrm{QR}_{0} \mathfrak{C} & \text { if } X \in \mathfrak{X}^{\prime} .\end{cases}
$$

In particular, $\mathfrak{C}$ is $\mathfrak{X}$-saturated if and only if $\overline{\mathfrak{C}}_{\mathfrak{X}}=\mathfrak{C}$, and if $\mathfrak{X}_{1}$ and $\mathfrak{X}_{2}$ are two classes of groups such that $\mathfrak{X}_{1} \subseteq \mathfrak{X}_{2}$, then every $\mathfrak{X}_{2}$-saturated formation is also $\mathfrak{X}_{1}$-saturated.

\section{RELATION BETWEEN $\omega$-SATURATED formations AND $\mathfrak{X}$-SATURATED FORMATIONS}

Let $\omega$ be a set of primes. A formation $\mathfrak{F}$ is said to be $\omega$-saturated if it is $p$-saturated for every prime $p \in \omega$. As it is proved in [12], it is possible to characterise $\omega$-saturated formations by means of some $\omega$-local definitions.

The main goal of this section is to study the relation between $\omega$-saturated formations and $\mathfrak{X}$-saturated formations.

Suppose that $\mathfrak{F}$ is an $\omega$-saturated formation. Then if $\mathfrak{X}_{\omega}$ is the class of all simple $\omega$-groups, we have that $\mathrm{O}_{\mathfrak{X}_{\omega}}(G)=\mathrm{O}_{\omega}(G)$ for each group $G$ and a group $G$ belongs to $\mathfrak{F}$ if and only if $G /\left(\Phi(G) \cap \mathrm{O}_{\omega}(G)\right) \in \mathfrak{F}$. Consequently since $\Phi_{x_{\omega}}(A) \leqslant \Phi(A) \cap \mathrm{O}_{\omega}(A)$ for every group $A$, it follows that $\mathfrak{F}$ is $\mathfrak{X}_{\omega}$-saturated.

However, the family of $\mathfrak{X}_{\omega}$-saturated formations does not coincide with the one of $\omega$-saturated formations in general. This follows from the fact that there exist Baer formations which are not $\omega$-saturated for any $\omega \subseteq \mathbb{P}$.

EXAMPLE 1. Let us consider the formation $\mathfrak{F}:=\mathbb{E} \mathfrak{Y}$, where $\mathfrak{Y}:=\left(A_{n} \mid n \geqslant 5\right)$, that is, the formation of all finite groups whose composition factors are isomorphic to an alternating group of degree $n \geqslant 5$. It is clear that $\mathfrak{F}$ is a Baer formation. In particular, $\mathfrak{F}$ is $\mathfrak{X}$-saturated for every $\mathfrak{X} \subseteq \mathbb{P}$.

Assume that $\mathfrak{F}$ is $p$-saturated for a prime $p$. If $p \geqslant 5$, set $k:=p$; otherwise, set $k:=5$. As $p|| A_{k} \mid$, by $[4, \mathrm{~B}, 11.8]$ there exists a group $E$ with a normal elementary Abelian $p$ subgroup $A \neq 1$ such that $A \leqslant \Phi(E)$ and $E / A \cong A_{k}$. We have that $E /\left(\mathrm{O}_{p}(E) \cap \Phi(E)\right)$ $=E /\left(\mathrm{O}_{p}(E) \cap A\right)=E / A \in \mathfrak{F}$. Therefore $E \in \mathfrak{F}$, a contradiction. 
Therefore $\mathfrak{F}$ is not $\omega$-saturated for any set $\omega$ of primes. Moreover, by setting $\mathfrak{X}$ $:=\left(C_{2}\right)$ and $\omega:=\{2\}$, we have that $\mathfrak{F}$ is $\mathfrak{X}$-saturated, but not 2-saturated.

From the above discussion, the following question naturally arises:

Let $\omega \subseteq \mathbb{P}$. Is it possible to ensure the existence of a class $\mathfrak{X}(\omega)$ of simple groups such that char $\mathfrak{X}(\omega)=\pi(\mathfrak{X}(\omega))$ satisfying that a formation is $\omega$-saturated if and only if it is $\mathfrak{X}(\omega)$-saturated?

The following example shows that the answer is negative.

EXAMPLE 2. Consider the formation

$$
\mathfrak{F}:=\left(G \mid \text { all Abelian composition factors of } G \text { are isomorphic to } C_{2}\right) .
$$

Suppose that $\mathfrak{F}$ is $\mathfrak{X}$-saturated for a class $\mathfrak{X}$ containing a non-abelian simple group $E$ and $\pi(\mathfrak{X})=$ char $\mathfrak{X}$. There exists a prime $p \neq 2$ dividing the order of $E$. Hence $p \in \mathfrak{X}$. Since $E \in \mathfrak{F}$, it follows that $f(p) \neq \emptyset$. Therefore $C_{p} \in \mathfrak{F}$, a contradiction.

Since $\mathfrak{F}$ is solubly saturated, we have that $\mathfrak{F}$ is $\mathfrak{X}$-saturated exactly for the classes $\mathfrak{X}$ such that $\mathfrak{X} \subseteq \mathbb{P}$. Since $\mathfrak{F}$ is clearly 2 -saturated, if we assume the existence of a class $\mathfrak{X}(2)$ fulfilling the property, it follows that $\mathfrak{X}(2) \subseteq \mathbb{P}$. This is not possible, as shown in Example 1.

However, inside the $\omega$-separable universe, the situation is clearer.

THEOREM 3. Let $\omega$ be a set of primes and let $\mathfrak{X}_{\omega}$ be the class of all simple $\omega$ groups. If $\mathfrak{F}$ is an $\mathfrak{X}_{\omega}$-saturated formation composed of $\omega$-separable groups, then $\mathfrak{F}$ is $\omega$-saturated.

Proof: Let $\mathfrak{X}=\mathfrak{X}_{\omega}$ be the class of all simple $\omega$-groups. We know that $\mathfrak{F}=\operatorname{LF}_{\mathfrak{X}}(f)$, where

$$
f(E)= \begin{cases}\mathrm{Q} \mathrm{R}_{0}\left(G / \mathrm{C}_{G}(H / K) \mid G \in \mathfrak{F}, H / K \text { is an } \mathfrak{X}_{p} \text {-chief factor of } G\right) \\ \mathfrak{F} \quad \text { if } E=p \in \omega, \\ \text { if } E \notin \mathfrak{X} .\end{cases}
$$

Let $G$ be a group of minimal order such that $G /\left(\Phi(G) \cap \mathrm{O}_{p}(G)\right) \in \mathfrak{F}$ but $G \notin \mathfrak{F}$ for some prime $p \in \omega$. Then $G$ has a unique minimal normal subgroup, $N$ say, such that $G / N \in \mathfrak{F}$ and $N \leqslant \Phi(G) \cap \mathrm{O}_{p}(G)$. Note that the intersection of the centralisers of the $\mathfrak{X}_{p}$-chief factors of $G$ is actually $\mathrm{O}_{p^{\prime}, p}(G)$, because $G$ is $\omega$-separable. Therefore $G / \mathrm{O}_{p^{\prime}, p}(G)$ $\epsilon f(p)$ because $\mathrm{O}_{p^{\prime}, p}(G / N)=\mathrm{O}_{p^{\prime}, p}(G) / N$ and $G / N \in \mathfrak{F}$. This implies that $G \in \mathfrak{F}$, a contradiction.

CoROLlaRY 2. Let $\mathfrak{F}$ be a formation composed of $\omega$-separable groups. Then $\mathfrak{F}$ is $\omega$-saturated if and only if $\mathfrak{F}$ is $\mathfrak{X}_{\omega}$-saturated, where $\mathfrak{X}_{\omega}$ is the class of all simple $\omega$-groups. 


\section{Proof of the MAIN RESUlT}

Proof of Theorem 1: Denote by form $(G)$ the formation generated by $G$, that is, the intersection of all formations containing $G$. It is known that $\mathfrak{N}$ form $(G)$, where $\mathfrak{N}$ is the class of all nilpotent groups, is a saturated formation. Therefore $\mathfrak{N}$ form $(G)$ is $\mathfrak{X}$-saturated and so $\mathfrak{H}$ is contained in $\mathfrak{N}$ form $(G)$.

Assume, arguing by contradiction, that $\mathfrak{F}$ is not $\mathfrak{X}$-saturated. Then there exists a group $A \notin \mathfrak{F}$ and a normal subgroup $N$ of $A$ such that $E=A / N \in \mathfrak{F}$ and $N \leqslant \Phi_{\mathfrak{X}}(A)$ $\leqslant \Phi(A) \cap \mathrm{O}_{\mathfrak{X}}(A)$.

STEP 1. For any group $1 \neq U \in \mathfrak{G}$, the group $(E \imath U)^{\mathfrak{G}}$ is not subdirectly contained in the base group of $E \imath U$, the regular wreath product of $E$ with $U$.

Let $1 \neq U \in \mathfrak{G}$ and denote $D=E \imath U$. Then $D^{\mathcal{B}}$ is contained in $K=E^{\natural}$, the base group of $D$. Suppose that $D^{\mathscr{C}}$ is subdirect in $K$. Then $D^{\mathfrak{G}} \in \mathfrak{F}$ because $K \in \mathfrak{F}$.

Consider now $D_{1}=A \backslash U$, and if $A_{0} \leqslant A$, we write $A_{0}^{\natural}=\left\{\left(a_{1}, \ldots, a_{|U|}\right) \mid a_{i}\right.$ $\left.\in A_{0}, \quad 1 \leqslant i \leqslant|U|\right\} \leqslant A^{\natural}=K_{1}$. Denote $R=N^{\natural}$. By $[4, \mathrm{~A}, 18.2]$, we have that $D_{1} / R$ is isomorphic to $D$. Assume that $\Phi_{\mathfrak{X}}(A)=\Phi\left(\mathrm{O}_{\mathfrak{X}}(A)\right)$. In this case, $\mathrm{O}_{\mathfrak{X}}\left(K_{1}\right)=\left(\mathrm{O}_{\mathfrak{X}}(A)\right)^{\natural}$ and $\Phi\left(\mathrm{O}_{\mathfrak{X}}\left(K_{1}\right)\right)=\left(\Phi\left(\mathrm{O}_{\mathfrak{X}}(A)\right)\right)^{\natural}$. This means that $R$ is contained in $\Phi_{\mathfrak{X}}\left(D_{1}\right)$.

Suppose now that $\Phi_{x}(A)=\Phi(A)$. Then there exists a prime $p$ such that $A$ $\in \mathrm{A}_{\mathfrak{X}_{p}}\left(\mathfrak{P}_{2}\right)$, that is, $A$ has an elementary Abelian normal $p$-subgroup $T$ such that $T \leqslant \Phi(A), A / T$ is primitive of type $2, \operatorname{Soc}(A / T) \in \mathrm{E} \mathfrak{X} \backslash \mathfrak{E}_{p^{\prime}}$ and $\mathrm{C}_{A}^{h}(T) \leqslant T$. Since $A / T$ is a primitive group of type 2 , it follows that $(A / T)<U \cong(A \imath U) / T^{\natural}=D_{1} / T^{\natural}$ is a primitive group of type 2 by $[4, \mathrm{~A}, 18.5]$. It is clear that $T^{\natural}$ is an elementary Abelian normal $p$-subgroup of $D_{1}$ contained in $\Phi\left(D_{1}\right)$. Moreover $\operatorname{Soc}\left(D_{1} / T^{\natural}\right) \cong \operatorname{Soc}(A / T)^{\natural}$. Therefore $\operatorname{Soc}\left(D_{1} / T^{\natural}\right) \in \mathrm{E} \mathfrak{X} \backslash \mathfrak{E}_{p^{\prime}}$. Assume that there exists $x \in D_{1}$ such that $x$ centralises all chief factors of $D_{1}$ below $T^{\natural}$. Let $H / K$ be a chief factor of $A$ below $T$ such that $H / K \notin \mathrm{Z}(A / K)$. Then $(H / K)^{\natural}$ is a chief factor of $D_{1}$ below $T^{\natural}$ and so $x$ centralises $(H / K)^{\mathrm{\natural}}$. In particular, each component of $x$ centralises the corresponding component of $(H / K)^{\natural}$. This implies that $x \in T^{\natural}$. Consequently $\mathrm{C}_{D_{1}}^{h}\left(T^{\natural}\right) \leqslant T^{\natural}$. Therefore we have proved that $D_{1} \in \mathrm{A}_{x_{p}}\left(\mathfrak{P}_{2}\right)$. In this case, $\Phi_{\mathfrak{x}}\left(D_{1}\right)=\Phi\left(D_{1}\right)$ and so $R \leqslant \Phi(A)^{\natural} \leqslant \Phi\left(D_{1}\right)$. Since $\mathfrak{H}$ is $\mathfrak{X}$-saturated, it follows that $D_{\mathfrak{1}} \in \mathfrak{H}$. This implies that $D_{1}^{\mathfrak{S}} \in \mathfrak{F}$. Since $D_{1} / R \cong D$, we have that $D^{\mathscr{G}}$ is isomorphic to $D_{1}^{\mathscr{E}} R / R$. It follows that $D_{1}^{\mathscr{O}}$ is subdirect in $K_{1}$. But hence $A$ is an epimorphic image of $D_{1}^{\mathscr{O}}$. Therefore $A \in \mathfrak{F}$, a contradiction.

STEP 2. If $C_{q}$ were the unique simple group in $\mathfrak{F}$ and $C_{q} \in \mathfrak{G}$, we would have $\mathfrak{S}_{q} \mathfrak{B}=\mathfrak{G}$ and $\mathfrak{H}=\mathfrak{G}$. This would be a contradiction if $q \in$ char $\mathfrak{X}$. Therefore there does not exist any prime $q \in$ char $\mathfrak{X}$ such that $\mathfrak{S}_{q}$ is contained in $\mathfrak{B}$.

First of all, assume that $C_{q}$ is the only simple group in $\mathfrak{F}, C_{q} \in \mathfrak{G}$, and that $\mathfrak{S}_{q} \mathfrak{G} \neq \mathfrak{B}$. Let $B$ be a group of minimal order in $\mathfrak{S}_{q} \mathfrak{G} \backslash \mathfrak{G}$. Hence $C=\operatorname{Soc}(B)$ is a minimal normal $q$-subgroup of $B$ and $1 \neq B / C \in \mathfrak{B}$.

Consider the group $D=E \imath(B / C)$. From Step 1, we know that $D^{\mathscr{G}}$ is not subdirect 
in the base group $K$ of $D$. Let $E_{1}$ be the first copy of $E$ in $K$ and let $F$ be the projection of $D^{\mathfrak{B}}$ in $E_{1}$. Then $F$ is a proper normal subgroup of $E_{1}$. Let $E_{0}$ be a maximal normal subgroup of $E_{1}$ such that $F \leqslant E_{0}$. Then $E_{1} / E_{0}$ is a simple group in $\mathfrak{F}$. Consequently $E_{1} / E_{0} \cong C_{q}$. It is clear that $K_{0}=E_{0}^{\natural}$ is a normal subgroup of $D$ and $D / K_{0} \cong C_{q} l(B / C)$ by $[4, \mathrm{~A}, 18.2]$. On the other hand, by [10, Lemma 1], we have that $\left.\left(E_{1} / F\right)\right\}(B / C)$ is a quotient of $D / D^{\mathfrak{B}}$. It follows then that $C_{q} \imath(B / C) \in \mathfrak{G}$. Therefore $C \imath(B / C)$ $\in \mathrm{R}_{0}\left(C_{q} \mathrm{l}(B / C)\right) \subseteq \mathfrak{G}$. Applying $[4, \mathrm{~A}, 18.9$ and $\mathrm{IV}, 1.14], B$ belongs to $\mathrm{QR}_{0}\left(C_{q} \mathrm{l}(B / C)\right)$ $\subseteq \mathfrak{G}$, a contradiction. Therefore $\mathfrak{S}_{q} \mathfrak{G}=\mathfrak{G}$. Let $G \in \mathfrak{H}$, and assume that $T=G^{\mathfrak{B}} \neq 1$. Hence $\mathrm{O}^{q}(T)<T$. Therefore $G / \mathrm{O}^{q}(T) \in \mathfrak{S}_{q} \mathfrak{G}=\mathfrak{G}$, a contradiction. It follows that $T=1$ and so $G \in \mathfrak{G}$. Consequently $\mathfrak{H}=\mathfrak{G}$ and so $\mathfrak{S}_{q} \mathfrak{H}=\mathfrak{S}_{q} \mathfrak{B}=\mathfrak{G}=\mathfrak{H}$, a contradiction if $q \in \operatorname{char} \mathfrak{X}$.

Assume now that there exists a prime $q \in$ char $\mathfrak{X}$ such that $\mathfrak{S}_{q} \subseteq \mathfrak{G}$. Since $\mathfrak{F} \neq(1)$, there exists a simple group $S \in \mathfrak{F}$. Assume $S \not \equiv C_{q}$. Let $G_{1}$ be an arbitrary $q$-group. Then $S \imath G_{1} \in \mathfrak{H} \subseteq \mathfrak{N}$ form $(G)$. If $S$ is not Abelian, then $S \imath G_{1} \in \operatorname{form}(G)$, and so $G_{1} \in$ form $(G)$. Assume now that $S$ is Abelian, then $S$ is isomorphic to $C_{r}$ for a prime $r \neq q$. Then $G_{1} \in$ form $(G)$. In both cases, $\mathfrak{S}_{q} \subseteq$ form $(G)$, a contradiction. Therefore $C_{q}$ is the only simple group in $\mathfrak{F}$ and the conclusion holds.

STEP 3. $G$ has a composition factor in $\mathfrak{X}$.

Denote by $\mathfrak{K}$ the class of composition factors of $G$ and assume that $\mathfrak{K} \cap \mathfrak{X}=\emptyset$. Consider the class $\mathrm{E} \mathfrak{K}$ of finite groups whose composition factors belong to $\mathfrak{K}$. Let $\mathfrak{L}$ be a formation contained in $E \mathfrak{K}$. Then it is rather easy to see that $\mathfrak{L}$ is $\mathfrak{X}$-saturated. Since form $(G) \subseteq \mathrm{E} \mathfrak{K}$ we have that form $(G)$ is $\mathfrak{X}$-saturated. Therefore $\mathfrak{F} \circ \mathfrak{G}=$ form $(G)$. By $[\mathbf{6}$, 4.5.8], it follows that $\mathfrak{F}$ consists of nilpotent groups. Since $\mathfrak{F}$ is subgroup-closed by [4, IV 1.16 , we have that $\mathfrak{F} \subseteq \mathfrak{H} \subseteq \mathrm{E} \mathfrak{K}$. It follows that $\mathfrak{F}$ is $\mathfrak{X}$-saturated, a contradiction.

STEP 4. Final contradiction.

Let $q$ be a prime dividing the order of a composition factor of $G$ in $\mathfrak{X}$. It follows that $\mathfrak{S}_{q} \subseteq \mathfrak{H}$ because $\mathfrak{H}$ is $\mathfrak{X}$-saturated. By Step 2, we have that $\mathfrak{S}_{q}$ is not contained in $\mathfrak{G}$. By $[\mathbf{2}$, Corollary $]$ and Step 2, we have that $\mathfrak{S}_{q} \subseteq \mathfrak{F}$. Moreover, by Step 1 and $[\mathbf{1 0}$, Lemma 3], there exists a prime $p$ such that $\mathfrak{S}_{p} \subseteq \mathrm{s}(\mathfrak{G})$, that is, given a group $P \in \mathfrak{S}_{p}$, there exists a group $G(P)$ in $\mathfrak{G}$ such that $P \leqslant G(P)$.

Assume that $p \neq q$. Consider $X_{P}=C_{q} \prec G(P) \in \mathfrak{H} \subseteq \mathfrak{N}$ form $(G)$. We have that $T_{P}=X_{P} / \mathrm{F}\left(X_{P}\right) \in$ form $(G)$. But $\mathrm{F}\left(X_{P}\right)$ is a $q$-group. It follows that form $(G)$ contains a group $T_{P}$ with a Sylow $p$-subgroup containing a copy of $P$. This is a contradiction. Therefore $p=q$.

On the other hand, by Step 2 we know that there exists a simple group $S$ in $\mathfrak{F}$ such that $S \not \approx C_{q}$. If $S$ is not Abelian, then $S \imath G(P) \in \mathfrak{F} \circ \mathfrak{G}=\mathfrak{H}$. Hence $S \nmid G(P) \in \mathfrak{N}$ form $(G)$, we have $G(P) \in$ form $(G)$, a contradiction. Therefore $S$ is cyclic, $S \cong C_{r}$ for a prime $r \neq q$. Let $Y=C_{r} \prec G(P)$. Then $Y \in \mathfrak{H} \subseteq \mathfrak{N}$ form $(G)$. Moreover, $\mathrm{F}(Y)$ is an $r$-group and $Y / \mathrm{F}(Y) \in \operatorname{form}(G)$. It follows that form $(G)$ contains a group $R_{P}$ with a Sylow 
$p$-subgroup containing a copy of $P$, final contradiction.

The same arguments to those used in the proof of Theorem 1 replacing $\Phi_{x}(G)$ by $\Phi(G) \cap \mathrm{O}_{p}(G)$ give an alternative proof of the result of Vishnevskaya for $p$-saturated formations. Moreover a second condition could be added to that result: $\mathfrak{B} \neq \mathfrak{S}_{p} \mathfrak{G}$. Taking into account that the $\omega$-saturated formations are exactly the $p$-saturated ones for all $p \in \omega$ the main result of [13] can be improved in the following way:

THEOREM 4. Let $\omega$ be set of primes. Let $\mathfrak{H}=\mathfrak{F} \circ \mathfrak{G}$ be an $\omega$-saturated formation generated by a group $G$. If $\mathfrak{F}$ and $\mathfrak{B}$ are non-trivial and either $\mathfrak{H} \neq \mathfrak{G}$ or $\mathfrak{S}_{p} \mathfrak{H} \neq \mathfrak{H}$ for all primes $p \in \omega$, then $\mathfrak{F}$ is $\omega$-saturated.

\section{REFERENCES}

[1] A. Ballester-Bolinches, C. Calvo and R. Esteban-Romero, 'On $\mathfrak{X}$-saturated formations of finite groups', preprint.

[2] A. Ballester-Bolinches and M.D. Pérez-Ramos, 'Some questions of the Kourovka Notebook concerning formation products', Comm. Algebra 26 (1998), 1581-1587.

[3] A. Ballester-Bolinches and L.A. Shemetkov, 'On lattices of $p$-local formations of finite groups', Math. Nachr. 186 (1997), 57-65.

[4] K. Doerk and T. Hawkes., Finite soluble groups, De Gruyter Expositions in Mathematics 4 (Walter de Gruyter, Berlin, New York, 1992).

[5] P. Förster, 'Projective Klassen endlicher Gruppen', Publ. Sec. Mat. Univ. Autònoma Barcelona 29 (1985), 39-76.

[6] W. Guo, The theory of classes of groups (Kluwer Academic Publishers, Dordrecht, 2000).

[7] V.D. Mazurov and E.I. Khukhro, editors, The Kourovka Notebook (unsolved problems in group theory), 12th edition (Russian Academy of Sciences, Siberian Division, Institute of Mathematics, Novosibirsk, 1992).

[8] V.D. Mazurov and E.I. Khukhro, editors, The Kourovka Notebook (unsolved problems in group theory), 14th edition (Russian Academy of Sciences, Siberian Division, Institute of Mathematics, Novosibirsk, 1999).

[9] L.A. Shemetkov, 'On the product of formations', Dokl. Akad. Nauk BSSR 28 (1984), 101-103.

[10] A.N. Skiba, 'On nontrivial factorisations of a one-generated local formation of finite groups', Contemp. Math. 131 (1992), 363-374.

[11] A.N. Skiba and L.A. Shemetkov, 'On partially local formations', Dokl. Akad. Nauk Belarusi 39 (1995), 9-11.

[12] A.N. Skiba and L.A. Shemetkov, 'Multiply $\omega$-local formations and Fitting classes of finite groups', Siberian Adv. Math. 10 (2000), 1-30.

[13] T.R. Vishnevskaya, 'On factorizations of one-generated p-local formations', Izv. Gomel. Gos. Univ. Im. F. Skoriny Vopr. Algebry 3 (2000), 88-92. 
Departament d'Àlgebra Universitat de València

Dr. Moliner, 50

E-46100 Burjassot

València

Spain

e-mail: Adolfo.Ballester@uv.es

clacalo@alumni.uv.es
Departament de Matemàtica Aplicada Universitat Politècnica de València

Camí de Vera, $\mathbf{s} / \mathbf{n}$

E-46022 València

Spain

e-mail: resteban@mat.upv.es 